\title{
Alteration of synaptic plasticity in rat dorsal striatum induced by chronic ethanol intake and withdrawal via ERK pathway
}

\author{
Sheng-zhong $\mathrm{CUI}^{1}$, Shen-jun WANG ${ }^{1}$, Jing $\mathrm{LI}^{2}$, Gui-qin $\mathrm{XIE}^{1}$, Rong ZHOU ${ }^{1}$, Ling $\mathrm{CHEN}^{1}$, Xiao-ru YUAN ${ }^{1, *}$ \\ ${ }^{1}$ Department of Physiology, Nanjing Medical University, Nanjing 210029, China; ${ }^{2}$ Department of Pathophysiology, Nanjing Medical Uni- \\ versity, Nanjing 210029, China
}

\begin{abstract}
Aim: The dorsal striatum has been proposed to contribute to the formation of drug-seeking behaviors, leading to excessive and compulsive drug usage, such as addiction. The current study aimed to investigate the involvement of extracellular signal-regulated kinase (ERK) pathway in the modification of striatal synaptic plasticity.

Methods: Ethanol was administered to rats in drinking water at concentration of $6 \%(\mathrm{~V} / \mathrm{v})$ for 30 days. Rats were sacrificed on day 10 , 20 , or 30 during ethanol intake or on withdrawal day 1, 3, or 7 following 30-d ethanol intake. The striata were removed either for electrophysiological recording or for protein immuno-blot analysis. Extracellular recording technique was used to record population spikes (PS) induced by high-frequency stimulation (HFS) in the dorsolateral striatum (DLS).

Results: Corticostriatal long-term depression (LTD) was determined to be dependent upon ERK signaling. Chronic ethanol intake (CEI) attenuated ERK phosphorylation and LTD induction, whereas withdrawal for one day (W1D) potentiated ERK phosphorylation and LTD induction. These results showed that the impact of chronic ethanol intake and withdrawal on corticostriatal synaptic plasticity was associated with ethanol's effect on ERK phosphorylation. In particular, pharmacological inhibition of ERK hyper-phosphorylation by U0126 prevented LTD induction in the DLS and attenuated ethanol withdrawal syndrome as well.

Conclusion: In rat DLS, chronic ethanol intake and withdrawal altered LTD induction via ERK signaling pathway. Ethanol withdrawal syndrome is mediated, at least partly, by ERK hyper-phosphorylation in the DLS.
\end{abstract}

Keywords: long-term depression; ethanol; synaptic plasticity; dorsal striatum; extracellular signal-regulated kinase; U0126

Acta Pharmacologica Sinica (2011) 32: 175-181; doi: 10.1038/aps.2010.199

\section{Introduction}

Addiction is increasingly viewed as a pathological process of learning, involving cell signaling and synaptic mechanisms similar to those implicated in neural models of learning and memory ${ }^{[1-4]}$. Synaptic plasticity is required for neuroadaptations that result from a variety of environmental stimuli. Therefore, it is attractive to hypothesize that drug abuse causes long-term changes in behavior by altering synaptic function and plasticity in relevant brain circuits.

The involvement of the ventral striatum, or nucleus accumbens (NAc), in mediating drug reward and reinforcement is well established ${ }^{[2,4-6]}$. Recently, it has also been proposed that the dorsal striatum (caudate nucleus and putamen) is likely to be involved in advanced stages of addiction when drug use progresses toward a compulsive, habitual pathology ${ }^{[2,5,6]}$.

\footnotetext{
* To whom correspondence should be addressed.

E-mail xryuan@public1.ptt.js.cn

Received 2010-04-08 Accepted 2010-11-01
}

Previous work from our lab ${ }^{[7]}$ has shown that synaptic plasticity in the rat dorsal striatum was altered by chronic ethanol exposure and withdrawal. However, the relevant underlying molecular mechanism remains unclear.

It has been reported that extracellular signal-regulated kinase (ERK1/2, also known as p44 and p42 MAPK) signaling plays an important role in the induction of long-term potentiation (LTP). For example, stimuli inducing LTP in area CA1 of the hippocampus potently activate $\mathrm{ERK}^{[8]}$, whereas pharmacological inhibition of MEK, an upstream activator of ERK, inhibits $\operatorname{LTP}^{[8,9]}$. The participation of ERK in synaptic plasticity has also been found in other brain regions ${ }^{[10,11,12]}(e g$, LTD in the cerebellum and LTP in the visual cortex).

Furthermore, several studies ${ }^{[13-15]}$ have suggested that the ERK signaling pathway is one of the targets of ethanol. For example, acute application of ethanol has been shown to attenuate MAPK activation in cultured cortical neurons and in mouse cerebral cortex in vivo ${ }^{[13]}$. A reduction of MAPK activation by chronic intermittent ethanol treatment was 
observed in the amygdala, the cerebellum, the striatum and the hippocampus ${ }^{[14]}$.

Based on these results, we hypothesized that changes in ERK activation by ethanol may contribute to the modulation of corticostriatal plasticity following chronic ethanol intake (CEI) treatment. In the present study, we first investigated whether corticostriatal LTD, as indicated in other brain regions, was ERK dependent. We then compared the effects of CEI and withdrawal treatment on ERK activation and corticostriatal LTD induction. The results showed that the ERK signaling pathway was involved in both striatal LTD induction and the impairment of synaptic plasticity produced by CEI/withdrawal treatment.

\section{Materials and methods}

\section{Animals and drug application}

Male Sprague-Dawley rats (grade II) from the Experimental Animals Center of Jiangsu Province (Certification № 97001) weighing 150-170 g were housed in cages on a 12-h dark/ light cycle (light on at 7:00 am) with ad libitum access to food and water. Upon arrival in the laboratory, the animals were allowed to acclimate to the housing facilities for $3 \mathrm{~d}$ before use. All procedures were performed in accordance with the guidelines for the care and use of animals provided by the Experimental Animal Center of Jiangsu Province.

Ethanol was administered in drinking water at a concentration of $6 \%(v / v)$ for 30 consecutive days. Rats were sacrificed on day 10, 20 or 30 during ethanol intake or on withdrawal day 1,3 or 7 following 30 days of ethanol intake.

U0126, a selective inhibitor of MEK (ERK kinase), was dissolved in $1 \%$ dimethyl sulfoxide (DMSO) and delivered through intracerebroventricular (icv) injection $(100 \mu \mathrm{g} / \mathrm{rat}$ ) or bath application. DMSO was diluted with saline (sterilized $0.9 \%$ sodium chloride solution, $\mathrm{pH} 7.0$ ). In contrast to icv injection, a final DMSO concentration of $0.1 \%$, instead of $1 \%$, was used in bath application.

For icv injection, rats were anesthetized with pentobarbital sodium $(50 \mathrm{mg} / \mathrm{kg}$, ip). Guide cannulas ( $2.5 \mathrm{~mm}$ in length, 23 gauge) were implanted bi-ventricularly (A-P $-0.3 \mathrm{~mm}$ from bregma; $\mathrm{M}-\mathrm{L} \pm 1.2 \mathrm{~mm}$ from the midline) and anchored to the skull with stainless steel screws and dental cement. A wire stylet was inserted in the guide until microinjection was made. Freshly prepared reagents (U0126 or DMSO) were injected using a stepper-motorized micro-syringe (Stoelting, Wood Dale, IL, USA) at a rate of $1 \mu \mathrm{L} / \mathrm{min}$ (final volume $=5 \mu \mathrm{L} /$ side). The left half of the striata was used for phospho-ERK/ERK detection, whereas the right half of the striata was used for electrophysiological recording.

\section{Brain slice preparation}

Rats were anesthetized with halothane and killed by decapitation. The brains were then rapidly removed and cooled in ice-cold artificial cerebrospinal fluid (ACSF) containing (in mmol/L): $126 \mathrm{NaCl}, 2.5 \mathrm{KCl}, 1.2 \mathrm{NaH}_{2} \mathrm{PO}_{4}, 25 \mathrm{NaHCO}_{3}, 11$ D-glucose, $2.4 \mathrm{CaCl}_{2}$, and $1.2 \mathrm{MgCl}_{2}$ at $\mathrm{pH}$ 7.4. The brains were fixed to a silicone stage with cyanoacrylate. Coronal slices (400 $\mu \mathrm{m}$ thick) were cut with a manual vibratome (Campden Instruments, Loughborough, Leics, UK). The slices were immediately transferred to a holding chamber containing ACSF that was constantly oxygenated with $95 \% \mathrm{O}_{2}$ and $5 \% \mathrm{CO}_{2}$ and maintained at room temperature $22-25^{\circ} \mathrm{C}$ for $1-\mathrm{h}$ equilibration prior to recording. A hemi-slice containing cortex and striatum just anterior to the globus pallidus was then transferred to a recording chamber (TC-202A, Medical Systems Corp, NY), maintained at $32{ }^{\circ} \mathrm{C}$ and perfused with oxygenated $\operatorname{ACSF}\left(95 \% \mathrm{O}_{2}, 5 \% \mathrm{CO}_{2}\right)$ at a flow rate of $2 \mathrm{~mL} / \mathrm{min}$.

\section{Field potential recording}

Extracellular recording electrodes, pulled on a horizontal micropipette puller (Sutter P97; Sutter Instruments, Novato, CA, USA) and filled with $2 \mathrm{~mol} / \mathrm{L} \mathrm{NaCl}$ (electrode impedance 3-8 M $\Omega$ ), were placed within the striatum, located 1-2 $\mathrm{mm}$ ventral from the white matter, and set at the depth where maximal evoked population spike (PS) amplitude was observed. Field potentials were evoked by a constant current stimulus (50-200 $\mu \mathrm{A}, 100 \mu$ s duration) applied to the white matter via a glass-coated tungsten bipolar stimulating electrode placed dorsally with respect to the recording electrode. Baseline responses were set to half-maximal amplitude. Stable responses were recorded for $20 \mathrm{~min}$ prior to high-frequency stimulation (HFS; four bursts consisting of 100 pulses at 100 $\mathrm{Hz}$ delivered every $20 \mathrm{~s}$ ). Stimulus intensity was adjusted to a level evoking maximal response during HFS.

The majority of the slices were also subjected to maximal stimulation at the end of the experiment to ensure that no significant deterioration of the slices had occurred during the experiment, as determined by the presence of a maximal PS in response to such stimulation.

Data were filtered (high-pass, $0.1 \mathrm{~Hz}$; low-pass $3 \mathrm{kHz}$ ), amplified and digitized using an Axoclamp 2B amplifier and Digidata 1200 interface (Axon Instruments, CA), and then stored in a computer by pClamp 6.0 software and analyzed using Clampfit software.

\section{Western blot assays}

Dorsolateral striata were micro-dissected on ice using established anatomical landmarks. They were then immediately frozen in liquid nitrogen and stored in a $-80^{\circ} \mathrm{C}$ freezer until use. For experiments, all of the samples were homogenized in lysis buffer containing the following: $50 \mathrm{mmol} / \mathrm{L}$ Tris- $\mathrm{HCl}$ (pH 7.5), $150 \mathrm{mmol} / \mathrm{L} \mathrm{NaCl}, 5 \mathrm{mmol} / \mathrm{L}$ EDTA, $10 \mathrm{mmol} /$ $\mathrm{L} \mathrm{NaF}, 1 \mathrm{mmol} / \mathrm{L}$ sodium orthovanadate, $1 \%$ TritonX-100, $0.5 \%$ sodium deoxycholate, $1 \mathrm{mmol} / \mathrm{L}$ phenylmethylsulfonyl fluoride and protease inhibitor cocktail (Complete; Roche, Mannheim, Germany). Protein extracts were quantified using BCA assay (Pierce, Rockford, IL, USA), normalized, size-fractionated by $10 \%$ SDS polyacrylamide gel electrophoresis ( 50 $\mu \mathrm{g} / \mathrm{lane}$ ) and transferred to nitrocellulose membranes. Then blots were blocked for $1 \mathrm{~h}$ with $5 \%$ bovine serum albumin or skim milk in $0.1 \%$ TBS-Tween at room temperature. Phosphorylated forms of ERK were determined by immunodetection with a specific antibody against Thr202 and Tyr204 phospho- 
rylation sites (Cell Signaling Tech, MA) at a dilution of 1:1000 in the same solution used for blocking. After incubation overnight at $4{ }^{\circ} \mathrm{C}$ with the primary antibody, blots were repeatedly washed in $0.1 \%$ TBS-Tween and incubated with a 1: 2000 dilution of a HRP-conjugated secondary antibody in $0.1 \%$ TBSTween for $1 \mathrm{~h}$. Following further washes and incubation with a chemiluminescent substrate (Pierce), the blots were exposed to Kodak Biomax MR film. Western blots probed for phosphorylated ERK were stripped in $62.5 \mathrm{mmol} / \mathrm{L}$ Tris- $\mathrm{HCl}(\mathrm{pH} 6.8)$, $2 \%$ SDS, and $100 \mathrm{mmol} / \mathrm{L} 2$-mercaptoethanol for $30 \mathrm{~min}$ at $50{ }^{\circ} \mathrm{C}$. As a control, the blots were then blocked and reprobed to detect total ERK (non-phosphorylated + phosphorylated). Autoradiograms were scanned and quantified using QuantityOne software.

\section{Data analysis}

LTD was defined as a stable decrease in field potential amplitude $(>15 \%) 40$ min post-HFS. The percentage change in field potential amplitude was determined by normalizing the mean response measured 50-60 min post-HFS to the mean response measured over the 10 -min period immediately prior to tetanic conditioning.

Statistical analyses were performed using ANOVA followed by Student-Newman-Keuls post-hoc test. To evaluate differences between two treatments, two-tailed Student's $t$-test comparison was performed. Values of $P<0.05$ were considered statistically significant. All values are expressed as means \pm SEM (unless otherwise stated).

\section{Results}

\section{Induction of corticostriatal LTD was blocked by U0126}

U0126 is a specific membrane-permeable inhibitor of MAPK kinase (MAPKK, also known as MEK), which phosphorylates and activates ERK1/2 $2^{[16]}$. To assess whether ERK activation plays a role in striatal synaptic plasticity, as has been suggested for other brain regions, U0126 was bath applied to the recording slices. Four rats, weighing $250 \pm 8.2 \mathrm{~g}$, were used in this experiment.

As shown in Figure 1A, Western blot analysis revealed that basal ERK phosphorylation in DLS was markedly reduced following 30-min bath application of $20 \mu \mathrm{mol} / \mathrm{L}$ U0126 (0.41 $\pm 0.08, n=4, P<0.05$ vs control), as we expected, whereas the basal ERK phosphorylation remained unchanged $(0.93 \pm 0.06$, $n=4$ ) following 30 -min bath application of $0.1 \%$ DMSO compared to control.

The baseline PS amplitude (mean responses of $10 \mathrm{~min}$ immediately prior to HFS) was $-0.8 \pm 0.15 \mathrm{mV}$ ( $n=8$ slices) in the control group, $-0.77 \pm 0.18 \mathrm{mV}$ ( $n=8$ slices) in the DMSO group, and $-0.75 \pm 0.14 \mathrm{mV}$ ( $n=7$ slices) in the U0126 group. There was no significant difference between baseline PS amplitude in the control group and DMSO/U0126 group, which indicates that bath application of either DMSO or U0126 had no direct effect on basal synaptic transmission in DLS.

In DMSO-treated slices, the mean PS amplitude, measured at 50-60 min post-HFS, decreased (relative to baseline) to $54.4 \% \pm 5.2 \%$ ( $n=8$ slices, Figure $1 \mathrm{~B}$ ), which was similar to that
A
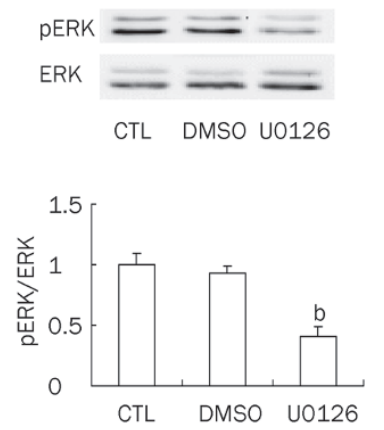

CTL
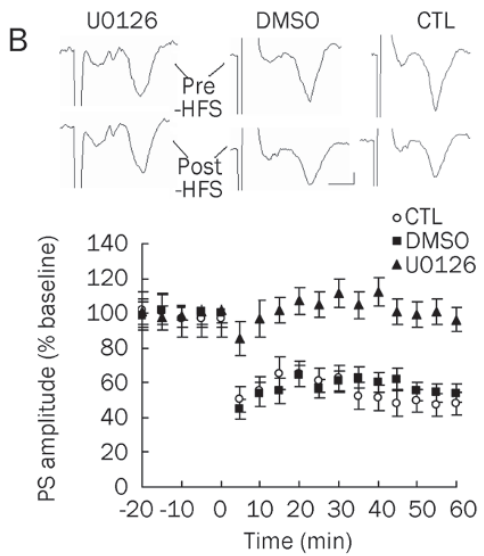

Figure 1. Effects of MEK inhibitor U0126 on striatal LTD induction and on ERK phosphorylation. (A) Thirty-minute bath application of $20 \mu \mathrm{mol} / \mathrm{L}$ U0126, but not $0.1 \%$ DMSO, inhibited ERK phosphorylation ( $n=4$ in each group) in rat DLS. (B) Mean PS (population spike) amplitudes, expressed as a percentage of the mean baseline value, in $20 \mu \mathrm{mol} / \mathrm{L}$ U0126-treated slices $(n=7)$, in $0.1 \%$ DMSO-treated slices $(n=8)$, and in control (CTL) slices $(n=8)$, respectively. $\mathrm{U} 0126$ or DMSO was present in the bath for $30 \mathrm{~min}$ before high-frequency stimulation (HFS). HFS was given at time 0 min. Upper panel: Sample traces of PS ( 5 min pre-HFS and 60 min post-HFS, respectively). Calibration: $2 \mathrm{~ms}, 0.2 \mathrm{mV}$. ${ }^{\mathrm{b}} \mathrm{P}<0.05$ vs control.

recorded in normal ACSF $(49.8 \% \pm 6.4 \%, n=8$ slices, Figure $1 B)$. This indicates that LTD can be reliably elicited in DMSOtreated slices, consistent with the above-mentioned results demonstrating that DMSO had no effect on ERK phosphorylation. In contrast, the mean PS amplitude post-HFS in U0126treated slices was $96.3 \% \pm 6.9 \%$ ( $n=7$ slices, Figure 1B) of baseline, suggesting that $30-\mathrm{min}$ bath application of $20 \mu \mathrm{mol} / \mathrm{L}$ U0126 abolished LTD induction.

These data demonstrated that striatal LTD is phospho-ERK dependent.

ERK phosphorylation was altered during chronic ethanol intake and withdrawal

Rats were divided into seven groups ( $n=5$ in each group): CTL (fed with tap water for 24 days), CEI10, CEI20, CEI30 (fed with $6 \%(v / v)$ ethanol for $10,20,30 \mathrm{~d}$, respectively), WD1, WD3, and WD7 (withdrawal for 1, 3, and $7 \mathrm{~d}$ after $30 \mathrm{~d}$ of ethanol intake). The body weights (mean \pm SD in $g$ ) of rats in each group were as follows: CEI10 (205.6 \pm 10.9$),$ CEI20 (245.2 \pm 11.3$)$, CTL (262.2 \pm 10.2$)$, CEI30 (297 \pm 10.5$),$ WD1 (304.4 \pm 11.9$),$ WD3 (318.2 \pm 14.8$)$, and WD7 (336.6 \pm 16.1$)$. Preliminary studies showed no significant differences in basal phospho- or total ERK protein levels within this weight range of rats.

Western blot results (Figure 2) showed that in DLS, chronic ethanol intake induced a decrease in ERK phosphorylation in the CEI10 $(0.54 \pm 0.05, P<0.05)$, CEI20 $(0.43 \pm 0.08, P<0.05)$ and CEI30 $(0.76 \pm 0.07, P<0.05)$ groups, respectively, in comparison with the CTL group. In contrast, phospho-ERK levels increased in the WD1 group $(1.32 \pm 0.08 ; P<0.05$ vs CTL) and showed no statistically significant changes in either the WD3 group $(0.96 \pm 0.10, n=5)$ or the WD7 group $(0.88 \pm 0.12, n=5)$. 

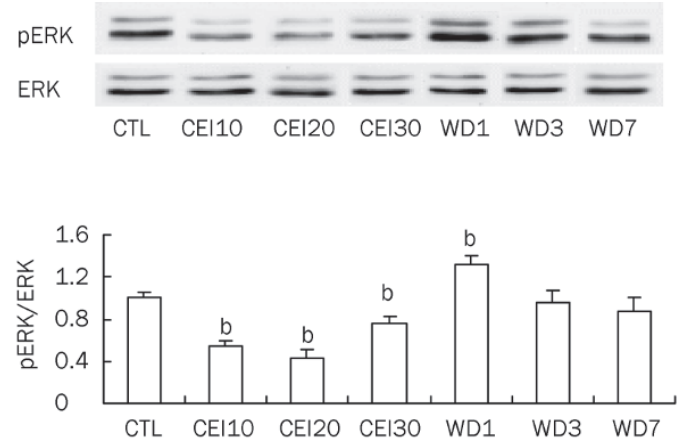

Figure 2. Chronic ethanol intake and withdrawal treatment changed phospho-ERK levels in rat DLS ( $n=5$ in each group). The upper panel is a representative Western blot. CTL, control; CEI10, CEI20, and CEI30 represent chronic ethanol intake for 10,20 , and 30 days, respectively. WD1, WD3, and WD7 represent chronic ethanol withdrawal for 1 day, 3 days, and 7 days, respectively, after 30 days of ethanol intake. ${ }^{\mathrm{b}} \mathrm{P}<0.05$ vs control.

These results indicate that in the DLS, chronic ethanol intake reduces ERK phosphorylation, whereas ethanol withdrawal enhances ERK phosphorylation transiently. Next, we determined whether chronic ethanol intake- and withdrawalinduced activities of ERK phosphorylation correlated with striatal LTD induction.

Changes of striatal LTD induction caused by chronic ethanol intake and withdrawal were associated with alterations in ERK phosphorylation

CEI10 (lower phospho-ERK) and WD1 (higher phosphoERK) groups were selected to determine whether changes in corticostriatal LTD induction would coincide with ERK phosphorylation following chronic ethanol intake or withdrawal.

U0126 or vehicle (1\% DMSO) was delivered through icv injection for 3 consecutive days prior to decapitation to manipulate ERK activity to verify the relationship between corticos- triatal synaptic plasticity and ERK phosphorylation. The pairfed rats, as the control group, received sham surgery.

As shown in Figure 3, using a tetanus stimulation (four bursts consisting of 100 pulses at $100 \mathrm{~Hz}$ delivered every $20 \mathrm{~s}$ ), LTD was readily induced in control brain slices (PS amplitudes post-HFS at $50.1 \% \pm 5.1 \%$ of baseline, $n=13$. Figure 3B). In the CEI10+DMSO groups, the PS amplitudes post-HFS increased to $81.2 \% \pm 4.4 \%$ of baseline $(n=11, P<0.05$ vs control), whereas phospho-ERK levels decreased to $0.58 \pm 0.11(n=5$, $P<0.05$ vs control; Figure $3 \mathrm{~A}$ ), which was consistent with the results shown in Figure 2. In the CEI10 + U0126 group, the PS amplitudes post-HFS increased to $101.7 \% \pm 7.8 \%$ of baseline ( $n=10, P<0.01$ vs control) along with a marked decrease in phospho-ERK levels $(0.31 \pm 0.08, n=5, P<0.01$ vs control). These data demonstrated an increase in the PS amplitudes post-HFS (in comparison with CTL) that accompanied a decrease in phospho-ERK levels in the CEI10 group or the CEI10+U0126 group, indicating that the attenuation or blockade of LTD induction might be related to changes in ERK phosphorylation.

In the WD1+DMSO group, phospho-ERK levels increased to $1.53 \pm 0.1$ of control $(n=5, P<0.05$ vs control; Figure $4 \mathrm{~A})$. However, no differences in the PS amplitudes post-HFS were found between the WD1+DMSO group and the CTL group (data not shown), as we would have expected.

This discrepancy could be accounted for the increased ERK phosphorylation as a result of HFS of corticostriatal fibers because corticostriatal fibers stimulate ERK signaling through the activation of glutamate receptors and an increase in intracellular calcium. In agreement with our hypothesis, when a weaker stimulation protocol (two bursts instead of four bursts) was utilized, a greater depression of the PS amplitudes post-HFS in WD1+DMSO groups $(34.5 \% \pm 4.8 \%$ of baseline vs $58.5 \% \pm 5.6 \%$ of baseline in CTL group, $P<0.05$, Figure $4 \mathrm{~B}$ ) was observed.

When U0126 was infused through icv injection in the WD1+U0126 group, the PS amplitudes post-HFS increased
A
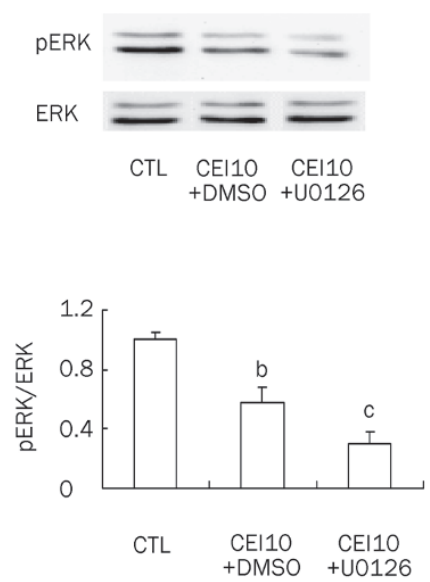

B

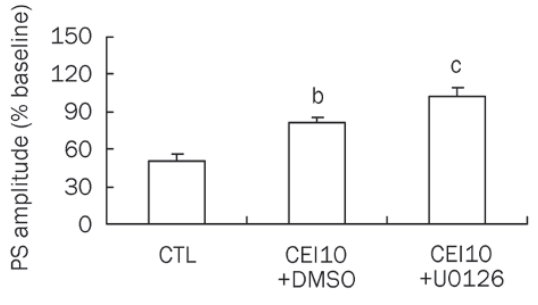

○ CTL

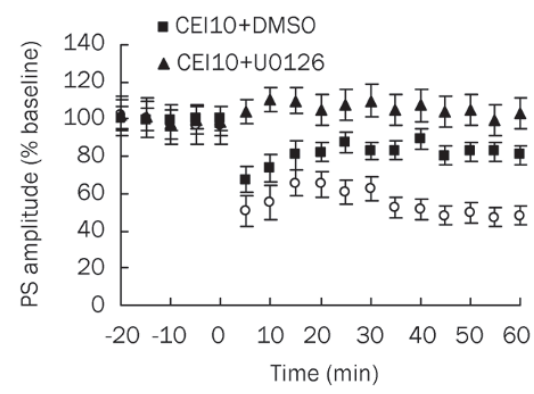

Figure 3. Ten days of chronic ethanol intake (CEI) decreased phospho-ERK levels and attenuated striatal LTD induction. MEK inhibitor U0126 enhanced the effects of CEI10 on both phospho-ERK levels and striatal LTD induction. (A) PhosphoERK levels decreased in both CEI10 and CEI10+U0126 groups ( $n=5$ in each group). (B) Induction of striatal LTD was attenuated in both CEI10 $(n=11)$ and CEI10+U0126 groups ( $n=10 ; n=13$ in CTL group). In the upper panel, the percentage change of PS amplitude is calculated by normalizing the mean response measured 50-60 min post-HFS to the mean response measured over the 10-min period immediately prior to HFS (baseline). In the lower panel, HFS was given at time 0 min. CTL, control; CEI10, 10 days of chronic ethanol intake. ${ }^{\mathrm{b}} P<0.05,{ }^{\mathrm{c}} P<0.01$ vs control. 


\section{A}
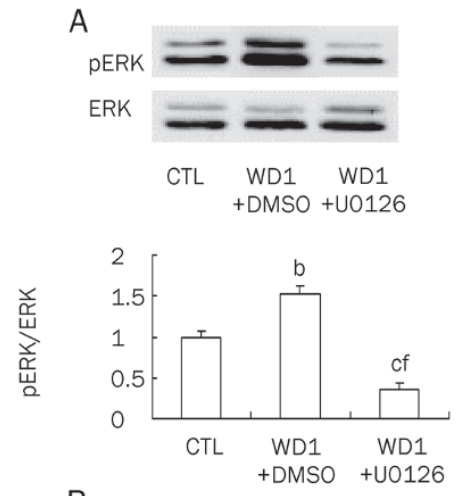

B
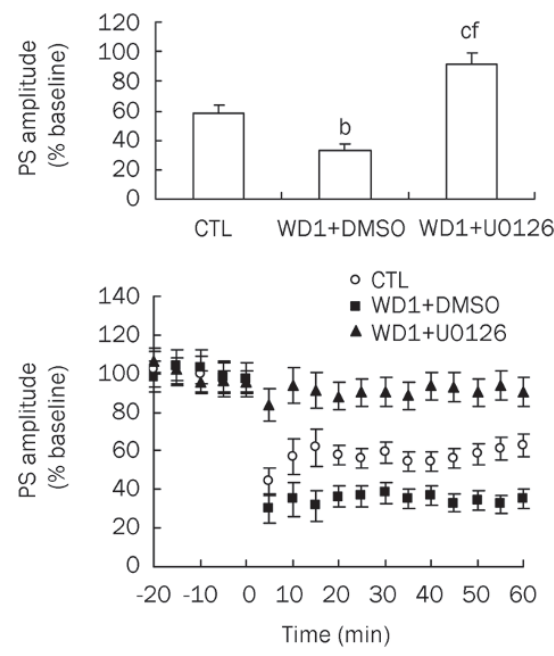

Figure 4. Effects of one-day withdrawal (WD1) and MEK inhibitor U0126 on both phospho-ERK levels and striatal LTD induction. (A) Phospho-ERK levels increased in WD1+DMSO group and decreased in WD1+U0126 group ( $n=5$ in each group). (B) Striatal LTD induction was potentiated by WD1+DMSO treatment $(n=10)$ and was blocked by WD1+U0126 treatment ( $n=11 ; n=14$ in CTL group). In the lower panel, HFS was given at time 0 min. CTL, control; WD1, 1-day withdrawal after 30 days of ethanol intake. ${ }^{b} P<0.05,{ }^{c} P<0.01$ vs control; ${ }^{f} P<0.01$ vs WD1+DMSO.

to $91.7 \% \pm 7.3 \%$ of baseline $(n=11, P<0.01$ vs control; $P<0.01$ vs WD1+DMSO, Figure 4A), whereas phospho-ERK levels decreased to $0.35 \pm 0.08$ ( $n=5, P<0.01$ vs control; $P<0.01$ vs WD1+DMSO, Figure 4B) of control, suggesting that LTD formation was inversely related to ERK activation.

Taken together, these results strongly indicated that chronic ethanol intake and withdrawal altered corticostriatal LTD induction via its effect on ERK phosphorylation in DLS.

\section{Ethanol withdrawal syndrome was attenuated by the infusion of U0126}

The ethanol withdrawal scores in both the WD1+DMSO group and the WD1+U0126 group were evaluated by an observer unaware of the drug treatment. The rating scale for the behavioral signs of ethanol withdrawal syndrome was used as described previously ${ }^{[7]}$. In congruence with the findings that icv infusion of U0126 can prevent both ERK hyper- phosphorylation and LTD induction in WD1 rats (Figure 4), ethanol withdrawal signs were also alleviated by the infusion of U0126, which is illustrated in Figure 5.

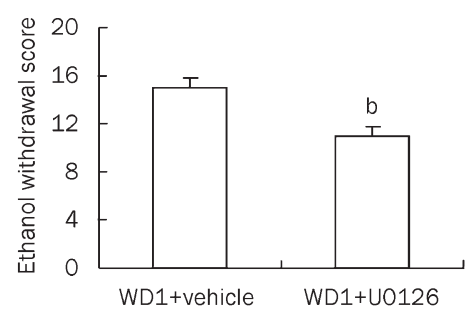

Figure 5. Ethanol withdrawal syndrome was attenuated by infusion of U0126 ( $n=5$ in each group). U0126 or vehicle (1\% DMSO) was delivered through icv injection for 3 consecutive days prior to evaluation. ${ }^{\mathrm{b}} \mathrm{P}<0.05$ vs WD1+vehicle.

\section{Discussion}

In the present study, we demonstrated that chronic ethanol intake decreased ERK phosphorylation, whereas ethanol withdrawal increased ERK phosphorylation transiently in rat DLS. Moreover, we showed that the alteration in corticostriatal synaptic plasticity induced by chronic ethanol intake and withdrawal occurred through the activation of ERK signaling pathway.

Both forms of long-lasting synaptic plasticity, LTP and LTD, can be induced by high-frequency synaptic stimulation of striatal neurons. In vivo studies demonstrated a preferential expression of LTP upon stimulation of cortical afferents ${ }^{[17,18]}$. In contrast, in striatal slices, LTD is the predominant form of plasticity observed following high-frequency afferent stimulation $^{[19-21]}$. LTP could only be induced in vitro using pharmacological manipulation, such as the blockade of dopamine receptors or the removal of extracellular magnesium ${ }^{[22]}$. Subsequent investigations delineated two intriguing factors that help to determine the direction of changes in synaptic efficacy at striatal synapses, namely the region of the striatum examined and the age of the animal ${ }^{[23,24]}$. Accordingly, LTP is preferentially expressed in dorsomedial striatum in younger rats under normal physiological conditions. This is consistent with the fact that synapses within the striatum develop along a lateralmedial gradient, with the synapses in lateral striatum maturing earlier than those in medial striatum ${ }^{[25]}$. Nevertheless, it is not surprising that LTD was invariably recorded in our experiment, given the use of older rats (2-3 months) and the recording subregion of striatum (DLS).

Although it has been previously shown that ERK phosphorylation is required for both $\operatorname{LTP}^{[9,10]}$ and $\operatorname{LTD}^{[26,11]}$ in hippocampal CA1 as well as LTD in the cerebellum ${ }^{[8]}$ and LTP in other cortical areas ${ }^{[12]}$, we provide the first evidence that ERK activation is required for striatal LTD induction.

The ethanol-induced modulation of ERK activity in vitro has been controversial, with potentiation reported by some ${ }^{[27,28]}$ and depression by others ${ }^{[29-31]}$. However, in vivo exposure data are more consistent, revealing an ethanol-induced decrease in 
ERK activation ${ }^{[13-15]}$, except in the report from Bachtell et al ${ }^{[32]}$. Our data are in agreement with previous reports in which chronic ethanol exposure in vivo decreased ERK phosphorylation. It should be noted that in CEI30 groups, the suppression of phospho-ERK levels was, while still significant, attenuated, which may reflect the adaptation or tolerance to long-term alcohol consumption. We also demonstrated an enhancement of ERK phosphorylation briefly after withdrawal ${ }^{[14]}$. This may be related to increased neural activity following disinhibition or hyperexcitability caused by ethanol withdrawal.

The mechanisms underlying the effects of chronic ethanol exposure on ERK phosphorylation are still under investigation. However, studies showing the activation of MAP kinase by $\mathrm{Ca}^{2+}$ via NMDA receptors or voltage-dependent $\mathrm{Ca}^{2+}$ channels have been very well documented ${ }^{[33-35]}$. Since ethanol inhibits both voltage-gated $\mathrm{Ca}^{2+}$ channels and NMDA receptor-associated $\mathrm{Ca}^{2+}$ influx ${ }^{[36-38]}$, it is possible that a decrease in the $\mathrm{Ca}^{2+}$ concentration in striatal neurons might decrease the phosphorylation of MAP kinase. Future studies will be required to identify the site and mechanism underlying the effects of ethanol on this pathway.

Anatomical studies have shown projections from the cortex to the medial striatum, including visual, auditory and limbic (ie, hippocampus, entorhinal and piriform cortices) afferents. In contrast, most cortical projections to the lateral striatum are of sensorimotor origin and, to a lesser degree, auditory and visual afferents ${ }^{[39]}$. Accordingly, recent studies suggest that the dorsomedial and dorsolateral striata have differential roles in different learning and memory paradigms. The dorsomedial striatum, in particular, has been shown to be critical for the learning of goal-directed actions. In contrast, the dorsolateral striatum appears to be involved in the formation of habits ${ }^{[40,41]}$. By showing that chronic ethanol intake and withdrawal differentially altered synaptic plasticity in dorsolateral striatum through ERK signaling pathway, our findings suggest that chronic alcohol abuse could disrupt the habit-formation process, and this neural maladaptation may consequently lead to habitual drug-seeking behavior.

To summarize, our current studies have shown that induction of corticostriatal LTD in rat is differentially altered by chronic ethanol intake and withdrawal, which occurs through ERK signaling pathway. Understanding the biochemical mechanism underlying chronic ethanol treatment-induced changes in striatal synaptic plasticity may aid in the development of more effective therapeutic agents for alcohol abuse.

\section{Acknowledgements}

This project was supported by the National Natural Science Foundation of China (No 30572173) and the Natural Science Foundation of the Education Committee of Jiangsu Province, China (№ 06KJB180071).

We thank Dr Shang SHEN at University of Nevada, Las Vegas, for critical reading of the manuscript.

\section{Author contribution}

Xiao-ru YUAN and Jing LI designed the research; Sheng- zhong CUI, Shen-jun WANG, Rong ZHOU, and Gui-qin XIE performed the research; Sheng-zhong CUI, Xiao-ru YUAN, and Ling CHEN analyzed the data and wrote the paper.

\section{References}

1 Nestler EJ. Molecular basis of long-term plasticity underlying addiction. Nat Rev Neurosci 2001; 2: 119-28.

2 Berke JD, Hyman SE. Addiction, dopamine, and the molecular mechanisms of memory. Neuron 2000; 25: 515-32.

3 Kelley AE. Memory and addiction: shared neural circuitry and molecular mechanisms. Neuron 2004; 44: 161-79.

4 Hyman SE, Malenka RC, Nestler EJ. Neural mechanisms of addiction: the role of reward-related learning and memory. Annu Rev Neurosci 2006; 29: 565-98.

5 Everitt BJ, Wolf ME. Psychomotor stimulant addiction: a neural systems perspective. J Neurosci 2002; 22: 3312-20.

6 Koob GF, Le Moal M. Drug addiction, dysregulation of reward, and alIostasis. Neuropsychopharmacology 2001; 24: 97-129.

7 Xia JX, Li J, Zhou R, Zhang XH, Ge YB, Yuan XR. Alterations of rat corticostriatal synaptic plasticity after chronic ethanol exposure and withdrawal. Alcohol Clin Exp Res 2006; 30: 819-24.

8 English JD, Sweatt JD. A requirement for the mitogen-activated protein kinase cascade in hippocampal long term potentiation. J Biol Chem 1997; 272: 19103-6.

9 Kanterewicz BI, Urban NN, McMahon DB, Norman ED, Giffen LJ, Favata MF, et al. The extracellular signal-regulated kinase cascade is required for NMDA receptor-independent LTP in area CA1 but not area CA3 of the hippocampus. J Neurosci 2000; 20: 3057-66.

10 Kawasaki H, Fujii H, Gotoh Y, Morooka T, Shimohama S, Nishida E, et al. Requirement for mitogen-activated protein kinase in cerebellar long-term depression. J Biol Chem 1999; 274: 13498-502.

11 Gallagher SM, Daly CA, Bear MF, Huber KM. Extracellular signal-regulated protein kinase activation is required for metabotropic glutamate receptor-dependent long-term depression in hippocampal area CA1. J Neurosci 2004; 24: 4859-64.

12 Di Cristo G, Berardi N, Cancedda L, Pizzorusso T, Putignano E, Ratto $\mathrm{GM}$, et al. Requirement of ERK activation for visual cortical plasticity. Science 2001; 292: 2337-40.

13 Kalluri HS, Ticku MK. Ethanol-mediated inhibition of mitogen-activated protein kinase phosphorylation in mouse brain. Eur J Pharmacol 2002; 439: 53-8.

14 Sanna PP, Simpson C, Lutjens R, Koob G. ERK regulation in chronic ethanol exposure and withdrawal. Brain Res 2002; 948: 186-91.

15 Roberto M, Nelson TE, Ur CL, Brunelli M, Sanna PP, Gruol DL. The transient depression of hippocampal CA1 LTP induced by chronic intermittent ethanol exposure is associated with an inhibition of the MAP kinase pathway. Eur J Neurosci 2003; 17: 1646-54.

16 Favata MF, Horiuchi KY, Manos EJ, Daulerio AJ, Stradley DA, Feeser WS, et al. Identification of a novel inhibitor of mitogen-activated protein kinase kinase. J Biol Chem 1998; 273: 18623-32.

17 Charpier S, Deniau JM. In vivo activity-dependent plasticity at corticostriatal connections: evidence for physiological long-term potentiation. Proc Natl Acad Sci USA 1997; 94: 7036-40.

18 Charpier S, Mahon S, Deniau JM. In vivo induction of striatal long-term potentiation by low-frequency stimulation of the cerebral cortex. Neurosci 1999; 91: 1209-22.

19 Calabresi P, Maj R, Pisani A, Mercuri NB, Bernardi G. Long-term synaptic depression in the striatum: physiological and pharmacological characterization. J Neurosci 1992; 12: 4224-33.

20 Lovinger DM, Tyler EC, Merritt A. Short- and long-term synaptic depres- 
sion in rat neostriatum. J Neurophysiol 1993; 70: 1937-49.

21 Wickens JR, Begg AJ, Arbuthnott GW. Dopamine reverses the depression of rat corticostriatal synapses which normally follows highfrequency stimulation of cortex in vitro. Neuroscience 1996; 70: 1-5.

22 Calabresi P, Pisani A, Mercuri NB, Bernardi G. Long-term potentiation in the striatum is unmasked by removing the voltage-dependent magnesium block of NMDA receptor channels. Eur J Neurosci 1992; 4: 929-35.

23 Smith R, Musleh W, Akopian G, Buckwalter G, Walsh JP. Regional differences in the expression of corticostriatal synaptic plasticity. Neuroscience 2001; 106: 95-101.

24 Partridge JG, Tang KC, Lovinger DM. Regional and postnatal heterogeneity of activity-dependent long-term changes in synaptic efficacy in the dorsal striatum. J Neurophysiol 2000; 84: 1422-9.

25 Butler AK, Uryu K, Chesselet M. A role for $N$-methyl- $D$-aspartate receptors in the regulation of synaptogenesis and expression of the polysialylated form of the neural cell adhesion molecule in the developing striatum. Dev Neurosci 1998; 20: 253-62.

26 Thiels E, Kanterewicz BI, Norman ED, Trzaskos JM, Klann E. Long-term depression in the adult hippocampus in vivo involves activation of extracellular signal-regulated kinase and phosphorylation of Elk-1. J Neurosci 2002; 22: 2054-62.

27 Roivainen R, Hundle B, Messing RO. Ethanol enhances growth factor activation of mitogen-activated protein kinases by a protein kinase Cdependent mechanism. Proc Natl Acad Sci USA 1995; 92: 1891-5.

28 Smith TL, Navratilova E. The effect of ethanol exposure on mitogenactivated protein kinase activity and expression in cultured rat astrocytes. Neurosci Lett 2003; 341: 91-4.

29 Hallak H, Seiler AE, Green JS, Henderson A, Ross BN, Rubin R. Inhibition of insulin-like growth factor-I signaling by ethanol in neuronal cells. Alcohol Clin Exp Res 2001; 25: 1058-64.

30 Ma W, Li BS, Maric D, Zhao WQ, Lin HJ, Zhang L, et al. Ethanol blocks both basic fibroblast growth factor- and carbachol-mediated neuroepi- thelial cell expansion with differential effects on carbachol-activated signaling pathways. Neuroscience 2003; 118: 37-47.

31 Seiler AE, Ross BN, Green JS, Rubin R. Differential effects of ethanol on insulin-like growth factor-I receptor signaling. Alcohol Clin Exp Res 2000; 24: 140-8.

32 Bachtell RK, Tsivkovskaia NO, Ryabinin AE. Alcohol-induced c-Fos expression in the Edinger-Westphal nucleus: pharmacological and signal transduction mechanisms. J Pharmacol Exp Ther 2002; 302: 516-24.

33 Orban CP, Chapman FP, Brambilla R. Is Ras-MAPK signaling pathway necessary for long term memory formation? Trends Neurosci 1999; 22: 38-44.

34 Rosen BL, Ginty DD, Weber JM, Greenberg EM. Membrane depolarization and calcium influx stimulate MEK and MAP kinase via activation of Ras. Neuron 1994; 12: 1207-21.

35 Xia Z, Dudek H, Miranti KC, Greenberg ME. Calcium influx via the NMDA receptor induces immediate early gene transcription by a MAP kinase/ERK-dependent mechanism. J Neurosci 1996; 16: 5425-36.

36 Lovinger DM, White G, Weight FF. Ethanol inhibits NMDA activated ion current in hippocampal neurons. Science 1989; 243: 1721-4.

37 Lovinger DM, White G, Weight FF. NMDA receptor-mediated synaptic excitation selectively inhibited by ethanol in hippocampal slice from adult rat. J Neurosci 1990; 10: 1372-9.

38 Walter HJ, Messing RO. Regulation of neuronal voltage-gated calcium channels by ethanol. Neurochem Int 1999; 35: 95-101.

39 McGeorge AJ, Faull RLM. The organization of the projection from the cerebral cortex to the striatum in the rat. Neuroscience 1989; 29 : 503-37.

40 Yin $\mathrm{HH}$, Knowlton BJ. The role of the basal ganglia in habit formation. Nature Rev Neurosci 2006; 7: 464-76.

41 Yin $\mathrm{HH}$, Knowlton BJ, Balleine BW. Lesions of dorsolateral striatum preserve outcome expectancy but disrupt habit formation in instrumental learning. Eur J Neurosci 2004; 19: 181-9. 